\title{
Some results for monotonically labelled simply generated trees
}

\author{
Bernhard Gittenberger ${ }^{1 \dagger}$ and Alois Panholzer ${ }^{1 \ddagger}$ \\ ${ }^{1}$ Institut für Diskrete Mathematik und Geometrie, Technische Universität Wien, Wiedner Hauptstr. 8-10/104, A-1040 \\ Wien, Austria. \{Bernhard.Gittenberger, Alois.Panholzer\}etuwien.ac.at
}

\begin{abstract}
We consider simply generated trees, where the nodes are equipped with weakly monotone labellings with elements of $\{1,2, \ldots, r\}$, for $r$ fixed. These tree families were introduced in Prodinger and Urbanek (1983) and studied further in Kirschenhofer (1984), Blieberger (1987), and Morris and Prodinger (2005). Here we give distributional results for several tree statistics (the depth of a random node, the ancestor-tree size and the Steiner-distance of $p$ randomly chosen nodes, the height of the $j$-st leaf, and the number of nodes with label $l$ ), which extend the existing results and also contain the corresponding results for unlabelled simply generated trees as the special case $r=1$.
\end{abstract}

Keywords: simply generated trees, monotone labellings, node depth, leaf height

\section{Introduction}

We consider rooted trees, where the nodes are labelled monotonically by elements of $\{1,2, \ldots, r\}$; this will mean that any sequence of labels lying on the direct path from the root to an arbitrary node in the tree is weakly monotone. Such tree structures were introduced in Prodinger and Urbanek (1983) and studied further in Kirschenhofer (1984) and Blieberger (1987). Very recently some parameters in monotonically labelled trees are treated in Morris and Prodinger (2005). These tree structures appear in the context of evaluating arithmetic expressions via the corresponding expression trees, see Blieberger (1987).

The previous work deals either with $t$-ary trees, Motzkin trees (unary-binary trees) or with ordered trees (planted plane trees) as the underlying model for the non-labelled trees, which are then equipped with monotone labelling with elements of $\{1,2, \ldots, r\} .^{\S}$ In Prodinger and Urbanek (1983) and Blieberger (1987) asymptotic results for the number $T_{n}^{[r]}$ of monotonically labelled trees with elements of $\{1, \ldots, r\}$ of size $n$ are obtained. In Kirschenhofer (1984) first results for the shape of such trees are given: he gives asymptotic equivalents for the expectation $\mathbb{E}\left(H_{n, j}\right)$ for $j$ fixed and $n \rightarrow \infty$, where $H_{n, j}$ measures the height (counted by the number of edges lying on the direct path from the root) of the $j$-st leaf (who are enumerated from left to right) in a random size- $n$ tree. Morris and Prodinger (2005) use the method of moments to obtain limiting distribution results for the Steiner-distance $Y_{n, p}$ and the ancestor-tree size $X_{n, p}$ of $p$ randomly chosen nodes in a random size- $n$ monotonically labelled binary tree (for $p$ fixed and $n \rightarrow \infty)$. For $t$-ary trees and ordered trees they give asymptotic equivalents for the first two moments. The size of the ancestor-tree of $p$ chosen nodes $v_{1}, \ldots, v_{p}$ in a rooted tree measures the size of the tree spanned by the root and $v_{1}, \ldots, v_{p}$ and therefore counts the number of nodes that are lying on at least one direct path from the root to $v_{i}$ for $1 \leq i \leq p$, and the Steiner-distance of $p$ chosen nodes $v_{1}, \ldots, v_{p}$ in a tree counts the number of nodes that are lying on at least one direct path from $v_{i}$ to $v_{j}$ for $1 \leq i \leq j \leq p$. The special instance $p=1$ of $X_{n, p}$ measures the depth $D_{n}$ of a random node in a random size- $n$ tree and is of particular interest.

In the present work we use as the underlying non-labelled tree model so called simply generated tree families, where of course $t$-ary trees and ordered trees are included as the most prominent members. We are studying then for monotonically labelled simply generated trees the tree statistics mentioned above,

\footnotetext{
†This author's work has been partially supported by the Austrian Science Foundation FWF, grant P16053-N05.

¥ This work is partially supported by the ÖAD, grant 11/2005, and by the Austrian Science Foundation FWF, grant P18009-N12.

$\S$ In Prodinger and Urbanek (1983) also monotonically labelled rooted trees (also called Pólya trees) are studied, where an asymptotic equivalent for the number of such trees with $n$ nodes is given. For the tree parameters studied here we will not treat this tree model, since such a distributional analysis is not even done for the unlabelled case $r=1$.
} 
which extend the previous work on this subject. In particular we will give limiting distribution results for the random variables $D_{n}, X_{n, p}, Y_{n, p}$ and $H_{n, j}$ for $n \rightarrow \infty$ and $p$ fixed resp. $j \sim c \kappa_{r} n$, with $0<c<1$. Here $\kappa_{r}$ is the constant (depending on the tree family considered), which appears in $\mathbb{E}\left(L_{n}\right) \sim \kappa_{r} n$, where $L_{n}$ is the random variable that counts the number of leaves in a random monotonically labelled tree with elements of $\{1, \ldots, r\}$ of size $n$. The corresponding results for ordinary (= unlabelled) simply generated trees (which are all well known, see Drmota (1994); Meir and Moon (1978); Panholzer (2004)) are of course always contained as the special case $r=1$.

Furthermore we show that the number of nodes $N_{n}^{[l]}$ labelled with $l$, with $1 \leq l \leq r$, in a random monotonically labelled size- $n$ tree with elements of $\{1, \ldots, r\}$, are for $r \geq 2$ asymptotically Gaussian distributed.

It is planned by the authors to continue the present work and possibly establish functional limit laws as obtained for simply generated trees (see e. g. Gittenberger (1999)).

\section{Preliminaries}

A family $\mathcal{T}_{r}$ of monotonically labelled simply generated trees with elements of $\{1, \ldots, r\}$ can be defined (in analogy to the definition of Meir and Moon (1978) of simply generated tree families) in the following way. A sequence of non-negative numbers $\left(\varphi_{k}\right)_{k \geq 0}$ with $\varphi_{0}>0\left(\varphi_{k}\right.$ can be seen as the multiplicative weight of a node with out-degree $k$ ) is used to define the weight $w(T)$ of any ordered tree (= planted plane tree) $T$ by $w(T)=\prod_{v} \varphi_{d(v)}$, where $v$ ranges over all vertices of $T$ and $d(v)$ is the out-degree of $v$. Since we want to exclude degenerate cases we always assume that there exists a $k \geq 2$ such that $\varphi_{k}>0$. Furthermore, $\mathcal{L}^{[r]}(T)$ denotes the set of different monotone labellings of the tree $T$ with elements of $\{1,2, \ldots, r\}$ and $L^{[r]}(T):=\left|\mathcal{L}^{[r]}(T)\right|$ its cardinality. Then the family $\mathcal{T}_{r}$ consists of all trees $T$ together with their weights $w(T)$ and the set of monotone labellings $\mathcal{L}^{[r]}(T)$. For brevity we will call a family $\mathcal{T}$ of trees $M$-labelled, if it consists of simply generated trees labelled monotonically with elements in $M$.

For a given degree-weight sequence $\left(\varphi_{k}\right)_{k \geq 0}$, we define the total weights

$$
T_{n}^{[r]}:=\sum_{|T|=n} w(T) \cdot L^{[r]}(T),
$$

where $|T|$ denotes the size of the tree $T$. For integer sequences $\left(\varphi_{k}\right)_{k>0}$, the quantities $T_{n}^{[r]}$ can be considered as the number of different $\{1, \ldots, r\}$-labelled size- $n$ trees of $\mathcal{T}_{r}$.

Furthermore we define by

$$
\varphi(t):=\sum_{k \geq 0} \varphi_{k} t^{k}
$$

the degree-weight generating function $\varphi(t)$, which contains all the information required for analysing the tree parameters considered here.

However it is more instructive to define the tree families $\mathcal{T}_{r}$ by systems of formal equations as done for $t$-ary trees and ordered trees in Prodinger and Urbanek (1983). To do this we use the auxiliary families $\tilde{\mathcal{T}}_{r}$ of $\{2,3, \ldots, r+1\}$-labelled trees. Then $\mathcal{T}_{r}$ can be described by the system of formal recurrences

$$
\begin{aligned}
& \mathcal{T}_{1}=11 \times \varphi\left(\mathcal{T}_{1}\right), \\
& \mathcal{T}_{2}=(1) \times \varphi\left(\mathcal{T}_{2}\right)+\tilde{\mathcal{T}}_{1}, \\
& \quad \vdots \\
& \mathcal{T}_{r}=11 \times \varphi\left(\mathcal{T}_{r}\right)+\tilde{\mathcal{T}}_{r-1},
\end{aligned}
$$

with $\bigcirc$ a node and $\varphi\left(\mathcal{T}_{i}\right)$ a substituted structure.

This formal equation (1) can be translated directly into the following system of functional equations for the generating functions $T_{r}(z):=\sum_{n \geq 1} T_{n}^{[r]} z^{n}$ (of course, the corresponding generating functions for $\mathcal{T}_{r}$ and $\tilde{\mathcal{T}}_{r}$ coincide):

$$
\begin{aligned}
T_{1}(z) & =z \varphi\left(T_{1}(z)\right), \\
T_{2}(z) & =z \varphi\left(T_{2}(z)\right)+T_{1}(z), \\
& \vdots \\
T_{r}(z) & =z \varphi\left(T_{r}(z)\right)+T_{r-1}(z) .
\end{aligned}
$$


Assumption 1 For our further analysis we always make the following assumptions on the degree-weight generating function $\varphi(t)$ :

(i) $\varphi(t)$ is aperiodic, i. e. $\operatorname{gcd}\left\{k: \varphi_{k}>0\right\}=1$,

(ii) $\varphi(t)$ has a positive radius of convergence $R>0$,

(iii) For all $r \geq 1$ exists a minimal positive solution $\tau_{r}<R$ of the equation

$$
t=\frac{\varphi(t)}{\varphi^{\prime}(t)}+T_{r-1}\left(\frac{1}{\varphi^{\prime}(t)}\right)
$$

Of course, one could also treat $\varphi(t)$ with period $d:=\operatorname{gcd}\left\{k: \varphi_{k}>0\right\}>1$ analogous to (unlabelled) simply generated tree families, but we restrict ourselves to this case. Although it seems hard to verify assumption (iii) in general, one can give sufficient conditions that cover the interesting cases. E. g., assumption (iii) always holds for entire degree-weight generating functions $\varphi(t)$ (as in the instance of polynomials, or equivalently for trees with bounded degrees). Moreover, for functions $\varphi(t)$ with a finite radius of convergence $R<\infty$, assumption (iii) holds if $\lim _{t \rightarrow R^{-}} \frac{\varphi(t)}{\varphi^{\prime}(t)}=0$ (as in the instance of ordered trees).

With Assumption 1 it follows then with arguments as in Meir and Moon (1978) or Drmota (2004) that the unique dominant singularity $\rho_{r}$ of $T_{r}(z)$ is given by $\rho_{r}:=\frac{1}{\varphi^{\prime}\left(\tau_{r}\right)}$, where $\tau_{r}$ is defined above. It is easily seen that $\tau_{1}<\tau_{2}<\tau_{3}<\ldots$, whereas $\rho_{1}>\rho_{2}>\rho_{3}>\ldots$

The local expansion of $T_{r}(z)$ around the dominant singularity $z=\rho_{r}$ follows also directly from Drmota (2004):

$$
T_{r}(z)=g_{r}(z)-h_{r}(z) \sqrt{1-\frac{z}{\rho_{r}}}=\tau_{r}-\sqrt{\frac{2\left(\varphi\left(\tau_{r}\right)+T_{r-1}^{\prime}\left(\rho_{r}\right)\right)}{\varphi^{\prime \prime}\left(\tau_{r}\right)}} \sqrt{1-\frac{z}{\rho_{r}}}+\mathcal{O}\left(1-\frac{z}{\rho_{r}}\right),
$$

where $g_{r}(z)$ and $h_{r}(z)$ are analytic functions in a neighbourhood of $z=\rho_{r}$.

Singularity analysis (Flajolet and Odlyzko (1990)) gives thus the following asymptotic expansion of the number $T_{n}^{[r]}$ of $\{1, \ldots, r\}$-labelled trees of size $n$ :

$$
T_{n}^{[r]}=\sqrt{\frac{\varphi\left(\tau_{r}\right)+T_{r-1}^{\prime}\left(\rho_{r}\right)}{2 \pi \varphi^{\prime \prime}\left(\tau_{r}\right)}} \rho_{r}^{-n} n^{-\frac{3}{2}}\left(1+\mathcal{O}\left(n^{-1}\right)\right) .
$$

For the most interesting cases of $t$-ary trees and ordered trees an asymptotic equivalent for $T_{n}^{[r]}$ was already established in Prodinger and Urbanek (1983), where also an asymptotic expansion of the singularities $\rho_{r}$ (for $r \rightarrow \infty$ ) was given.

\section{Results}

In this section we collect the results of the tree statistics considered, where we make in all theorems presented the assumptions given by Assumption 1. We further use the abbreviation $\sigma_{r}:=\sqrt{\rho_{r}^{2} \varphi^{\prime \prime}\left(\tau_{r}\right)\left(\varphi\left(\tau_{r}\right)+T_{r-1}^{\prime}\left(\rho_{r}\right)\right)}$ for a constant appearing frequently.

Theorem 1 The depth $D_{n}$ of a randomly chosen node in a random $\{1, \ldots, r\}$-labelled tree of size $n$ converges for $n \rightarrow \infty$ in distribution to a Rayleigh distributed random variable $X, \frac{D_{n}}{\sqrt{n}} \stackrel{(d)}{\longrightarrow} X$, with density functions $f(x)$ given by

$$
f(x)=\sigma_{r}^{2} x e^{-\frac{\sigma_{r}^{2} x^{2}}{2}}, \text { for } x \geq 0, \text { and } f(x)=0 \text { otherwise. }
$$

Theorem 2 The random variable $X_{n, p}$ which counts the size of the ancestor-tree of $p$ randomly chosen nodes in a random $\{1, \ldots, r\}$-labelled tree of size $n$ and the random variable $Y_{n, p}$ which counts the Steiner-distance of $p$ randomly chosen nodes in a random $\{1, \ldots, r\}$-labelled tree of size $n$, converge for fixed $p \geq 1$ (resp. $p \geq 2$ ), and $n \rightarrow \infty$ in distribution to generalized Gamma distributed random variables:

$$
\frac{X_{n, p}}{\sqrt{n}} \stackrel{(d)}{\longrightarrow} X_{p}, \quad \frac{Y_{n, p}}{\sqrt{n}} \stackrel{(d)}{\longrightarrow} Y_{p},
$$


where $X_{p}=Y_{p+1}$ and $Y_{p}$ is a random variable with density function

$$
f_{p}(x)=\frac{2}{(p-2) !}\left(\frac{\sigma_{r}}{\sqrt{2}}\right)^{2(p-1)} x^{2 p-3} e^{-\frac{\sigma_{r}^{2} x^{2}}{2}}, \text { for } x \geq 0, \text { and } f_{p}(x)=0 \text { otherwise. }
$$

If $g(a, h, A ; x)=\frac{|h|}{\Gamma(a) A}\left(\frac{x}{A}\right)^{a h-1} e^{-\left(\frac{x}{A}\right)^{h}}$ (for $x>0$ ) denotes the density function of the generalized Gamma distribution we get thus that $X_{p}$ resp. $Y_{p}$ have density functions $g\left(p, 2, \frac{\sqrt{2}}{\sigma_{r}} ; x\right)$ resp. $g(p-$ $\left.1,2, \frac{\sqrt{2}}{\sigma_{r}} ; x\right)$.

Theorem 3 The random variable $H_{n, j}$ which counts the height of the $j$-st (from left to right enumerated) leaf in a random $\{1, \ldots, r\}$-labelled tree of size $n$ converge in distribution for a (asymptotically) fixed ratio $\frac{j}{n}=c \kappa_{r}+o(1)$, with $0<c<1$, and $n \rightarrow \infty$ to a Maxwell distributed random variable $H_{c}$, $\frac{H_{n, j}}{\sqrt{n}} \stackrel{(d)}{\longrightarrow} H_{c}$, with density function $h_{c}(x)$ given by

$$
h_{c}(x)=\frac{\sigma_{r}^{3} x^{2}}{4 \sqrt{2 \pi}(c(1-c))^{\frac{3}{2}}} e^{-\frac{\sigma_{r}^{2} x^{2}}{8 c(1-c)}}, \text { for } x \geq 0, \text { and } h_{c}(x)=0 \text { otherwise. }
$$

The constant $\kappa_{r}$ appearing here is given by $\kappa_{r}=\frac{1}{\varphi\left(\tau_{r}\right)+T_{r-1}^{\prime}\left(\rho_{r}\right)} \sum_{l=1}^{r} \frac{\varphi_{0}}{\prod_{s=l}^{r-1}\left(1-\rho_{r} \varphi^{\prime}\left(T_{s}\left(\rho_{r}\right)\right)\right)}$.

Theorem 4 The random vector $\mathbf{N}_{n}=\left(N_{n}^{[1]}, \ldots, N_{n}^{[r]}\right)$, where $N_{n}^{[l]}$ counts the number of nodes that are labelled with element $l$ in a random $\{1, \ldots, r\}$-labelled tree (with $r \geq 2$ ) of size $n$, converges for $n \rightarrow \infty$ in distribution to a Gaussian distributed random vector with mean value $\sim n \mu$ and a certain covariance matrix $\sim n \Sigma:$

$$
\frac{\mathbf{N}_{n}-n \boldsymbol{\mu}}{\sqrt{n}} \stackrel{(d)}{\longrightarrow} \mathcal{N}(0, \boldsymbol{\Sigma})
$$

The mean vector $\boldsymbol{\mu}=\left(\mu^{[1]}, \ldots, \mu^{[r]}\right)$ is given by

$\mu^{[1]}=\frac{\varphi\left(\tau_{r}\right)}{\varphi\left(\tau_{r}\right)+T_{r-1}^{\prime}\left(\rho_{r}\right)}, \quad \mu^{[l]}=\frac{\varphi\left(T_{r+1-l}\left(\rho_{r}\right)\right)}{\varphi\left(\tau_{r}\right)+T_{r-1}^{\prime}\left(\rho_{r}\right)} \frac{1}{\prod_{s=r+1-l}^{r-1}\left(1-\rho_{r} \varphi^{\prime}\left(T_{s}\left(\rho_{r}\right)\right)\right)}, \quad$ for $2 \leq l \leq r$.

The proofs of these results are sketched in the next sections, where the following common abbreviations are used: $D_{x}$ is the differential operator w. r. t. $x, E_{x}$ denotes the evaluation operator at $x=1$, and $N_{x}$ denotes the evaluation operator at $x=0$.

\section{The depth of nodes}

We obtain from the formal description (1) of $\mathcal{T}_{r}$ the following system of functional equations for the generating functions $M_{r}(z, v):=\sum_{n \geq 1} \sum_{m \geq 0} n \mathbb{P}\left\{D_{n}=m\right\} T_{n}^{[r]} z^{n} v^{m}$ :

$$
\begin{aligned}
M_{1}(z, v) & =z v \varphi^{\prime}\left(T_{1}(z)\right) M_{1}(z, v)+z v \varphi\left(T_{1}(z)\right), \\
M_{2}(z, v) & =z v \varphi^{\prime}\left(T_{2}(z)\right) M_{2}(z, v)+z v \varphi\left(T_{2}(z)\right)+M_{1}(z, v), \\
& \vdots \\
M_{r}(z, v) & =z v \varphi^{\prime}\left(T_{r}(z)\right) M_{r}(z, v)+z v \varphi\left(T_{r}(z)\right)+M_{r-1}(z, v),
\end{aligned}
$$

which give the solutions

$$
M_{r}(z, v)=\frac{z v \varphi\left(T_{r}(z)\right)+M_{r-1}(z, v)}{1-z v \varphi^{\prime}\left(T_{r}(z)\right)} .
$$

To establish the limiting distribution of $D_{n}$ we use the method of moments and compute $E_{v} D_{v}^{s} M_{r}(z, v)$ for fixed integers $s \geq 1$. Using (6) one can show the following suitable expansion:

$$
E_{v} D_{v}^{s} M_{r}(z, v)=\frac{s !\left(z \varphi\left(T_{r}(z)\right)+M_{r-1}(z, 1)\right)}{\left(1-z \varphi^{\prime}\left(T_{r}(z)\right)\right)^{s+1}}+\mathcal{O}\left(\frac{1}{\left(1-z \varphi^{\prime}\left(T_{r}(z)\right)\right)^{s}}\right), \quad \text { for } s \geq 1
$$


Together with $M_{r}(z, 1)=z T_{r}^{\prime}(z)$, which follows from the definition, we get via (3) the following expansion around the dominant singularity $z=\rho_{r}$ :

$$
E_{v} D_{v}^{s} M_{r}(z, v)=\frac{s ! \rho_{r}\left(\varphi\left(\tau_{r}\right)+T_{r-1}^{\prime}\left(\rho_{r}\right)\right)}{\left(2 \rho_{r}^{2} \varphi^{\prime \prime}\left(\tau_{r}\right)\left(\varphi\left(\tau_{r}\right)+T_{r-1}^{\prime}\left(\rho_{r}\right)\right)\right)^{\frac{s+1}{2}}}\left(1-\frac{z}{\rho_{r}}\right)^{-\frac{s+1}{2}}+\mathcal{O}\left(\left(1-\frac{z}{\rho_{r}}\right)^{-\frac{s}{2}}\right) .
$$

Singularity analysis gives then

$$
\mathbb{E}\left(D_{n}^{s}\right) \sim \frac{\left[z^{n}\right] E_{v} D_{v}^{s} M_{r}(z, v)}{n\left[z^{n}\right] T_{r}(z)} \sim \frac{2^{\frac{s}{2}} \Gamma\left(\frac{s}{2}+1\right)}{\sigma_{r}^{s}} n^{\frac{s}{2}}
$$

Thus the $s$-th moment of the normalized depth $\frac{D_{n}}{\sqrt{n}}$ converges for $s \geq 1$ to the $s$-th moment of the Rayleigh distribution and together with the Theorem of Fréchet and Shohat this shows Theorem 1.

\section{Distances between nodes}

\subsection{Ancestor-tree size}

¿From the formal system (1) one gets the following system of functional equations for the generating functions $G_{r}(z, u, v):=\sum_{n \geq 1} \sum_{0 \leq p \leq n} \sum_{m \geq 0}\left(\begin{array}{l}n \\ p\end{array}\right) \mathbb{P}\left\{X_{n, p}=m\right\} T_{n}^{[r]} z^{n} u^{p} v^{m}$ (see Panholzer (2004) for the corresponding formula for simply generated trees, i. e. $r=1$, which can be extended easily):

$$
\begin{aligned}
G_{1}(z, u, v) & =z v(1+u) \varphi\left(G_{1}(z, u, v)\right)+(1-v) T_{1}(z), \\
G_{2}(z, u, v) & =z v(1+u) \varphi\left(G_{2}(z, u, v)\right)+(1-v) T_{2}(z)+G_{1}(z, u, v), \\
& \vdots \\
G_{r}(z, u, v) & =z v(1+u) \varphi\left(G_{r}(z, u, v)\right)+(1-v) T_{r}(z)+G_{r-1}(z, u, v) .
\end{aligned}
$$

We are interested here in the ancestor-tree size of $p \geq 1$ randomly chosen nodes for $p$ fixed. Thus we differentiate $G_{r}(z, u, v) p$-times w. r. t. $u$ and evaluate at $u=0$. Studying the resulting equations in a way analogous to Panholzer (2004) one can show inductively the following asymptotic equivalent (for $n \rightarrow \infty$, $m=\mathcal{O}(\sqrt{n})$ and $p \geq 1$ fixed):

$$
N_{u} D_{u}^{p} G_{r}(z, u, v) \sim \frac{(p-1) !}{\varphi^{\prime \prime}\left(T_{r}(z)\right) 2^{p-1}}\left(\begin{array}{c}
2(p-1) \\
p-1
\end{array}\right)\left(C_{r}(z) \varphi^{\prime \prime}\left(T_{r}(z)\right)\right)^{p} \frac{(z v)^{2 p-1}}{\left(1-z v \varphi^{\prime}\left(T_{r}(z)\right)\right)^{2 p-1}},
$$

with

$$
C_{r}(z)=\sum_{l=1}^{r} \frac{\left(\varphi^{\prime}\left(T_{r}(z)\right)\right)^{r-l} \varphi\left(T_{l}(z)\right)}{\prod_{s=l}^{r-1}\left(\varphi^{\prime}\left(T_{r}(z)\right)-\varphi^{\prime}\left(T_{s}(z)\right)\right)} .
$$

Extracting coefficients from (9) at $v^{m}$ immediately gives then

$$
\left[v^{m}\right] N_{u} D_{u}^{p} G_{r}(z, u, v) \sim \frac{m^{2 p-2}}{(p-1) !} \frac{\left(C_{r}(z) \varphi^{\prime \prime}\left(T_{r}(z)\right)\right)^{p}}{\varphi^{\prime \prime}\left(T_{r}(z)\right) 2^{p-1}\left(\varphi^{\prime}\left(T_{r}(z)\right)\right)^{2 p-1}}\left(z \varphi^{\prime}\left(T_{r}(z)\right)\right)^{m} .
$$

For the remaining task of extracting coefficients from (10) at $z^{n}$ we can use Cauchy's integration formula with a Hankel contour like integration path as was done for the corresponding parameter in simply generated trees in Panholzer (2004). Together with the evaluation $C_{r}\left(\rho_{r}\right)=\varphi\left(\tau_{r}\right)+T_{r-1}^{\prime}\left(\rho_{r}\right)$, which follows by induction, one gets finally the required asymptotic equivalent:

$$
\mathbb{P}\left\{X_{n, p}=m\right\}=\frac{\left[z^{n} v^{m}\right] N_{u} D_{u}^{p} G_{r}(z, u, v)}{p !\left(\begin{array}{l}
n \\
p
\end{array}\right)\left[z^{n}\right] T_{r}(z)} \sim \frac{2 m^{2 p-1}}{n^{p}(p-1) !}\left(\frac{\sigma_{r}}{\sqrt{2}}\right)^{2 p} e^{-\frac{\sigma_{r}^{2} m^{2}}{2 n}} .
$$

Setting $m=x \sqrt{n}+o(\sqrt{n})$ leads then to

$$
\sqrt{n} \mathbb{P}\left\{X_{n, p}=m\right\} \sim \frac{2}{(p-1) !}\left(\frac{\sigma_{r}}{\sqrt{2}}\right)^{2 p} x^{2 p-1} e^{-\frac{\sigma_{r}^{2} x^{2}}{2}} .
$$

Since the right-hand side of (11) is the density function of a generalized Gamma distribution the first part of Theorem 2 is shown. 


\subsection{Steiner-distance}

Since the parameters Steiner-distance and ancestor-tree size are closely related, we obtain that the generating functions $F_{r}(z, u, v):=\sum_{n \geq 1} \sum_{0<p<n} \sum_{m>0}\left(\begin{array}{l}n \\ p\end{array}\right) \mathbb{P}\left\{Y_{n, p}=m\right\} T_{n}^{[r]} z^{n} u^{p} v^{m}$ and $G_{r}(z, u, v)$ (as defined in Subsection 5.1) are connected; the formal equation (1) can be translated into the following system of equations (see again Panholzer (2004) for the corresponding formula for simply generated trees):

$$
\begin{aligned}
F_{1}(z, u, v)= & G_{1}(z, u, v)-z v \varphi^{\prime}\left(T_{1}(z)\right) G_{1}(z, u, v)+z \varphi^{\prime}\left(T_{1}(z)\right) F_{1}(z, u, v)-(1-v) z \varphi^{\prime}\left(T_{1}(z)\right) T_{1}(z), \\
F_{2}(z, u, v)= & G_{2}(z, u, v)-z v \varphi^{\prime}\left(T_{2}(z)\right) G_{2}(z, u, v)+z \varphi^{\prime}\left(T_{2}(z)\right) F_{2}(z, u, v)-(1-v) z \varphi^{\prime}\left(T_{2}(z)\right) T_{2}(z) \\
& +F_{1}(z, u, v)-G_{1}(z, u, v), \\
\vdots & \\
F_{r}(z, u, v)= & G_{r}(z, u, v)-z v \varphi^{\prime}\left(T_{r}(z)\right) G_{r}(z, u, v)+z \varphi^{\prime}\left(T_{r}(z)\right) F_{r}(z, u, v)-(1-v) z \varphi^{\prime}\left(T_{r}(z)\right) T_{r}(z) \\
& +F_{r-1}(z, u, v)-G_{r-1}(z, u, v) .
\end{aligned}
$$

We are here interested in the Steiner-distance for fixed $p \geq 2$. The task of computing $N_{u} D_{u}^{p} F_{r}(z, u, v)$ can be reduced to compute $N_{u} D_{u}^{p} G_{l}(z, u, v)$ for $1 \leq l \leq r$, since we get:

$N_{u} D_{u}^{p} F_{r}(z, u, v)=\frac{1-z v \varphi^{\prime}\left(T_{r}(z)\right)}{1-z \varphi^{\prime}\left(T_{r}(z)\right)} N_{u} D_{u}^{p} G_{r}(z, u, v)+\sum_{l=1}^{r-1} \frac{\prod_{s=l}^{r-1}\left(z(1-v) \varphi^{\prime}\left(T_{s}(z)\right)\right)}{\prod_{s=l}^{r}\left(1-z \varphi^{\prime}\left(T_{s}(z)\right)\right)} N_{u} D_{u}^{p} G_{l}(z, u, v)$.

It follows that (for $n \rightarrow \infty, m=\mathcal{O}(\sqrt{n})$ and $p \geq 2$ fixed):

$$
N_{u} D_{u}^{p} F_{r}(z, u, v) \sim \frac{1-z v \varphi^{\prime}\left(T_{r}(z)\right)}{1-z \varphi^{\prime}\left(T_{r}(z)\right)} N_{u} D_{u}^{p} G_{r}(z, u, v) .
$$

Together with the asymptotic equivalent (9) of $N_{u} D_{u}^{p} G_{r}(z, u, v)$ we can show the asymptotic equivalent here required:

$$
\mathbb{P}\left\{Y_{n, p}=m\right\}=\frac{\left[z^{n} v^{m}\right] N_{u} D_{u}^{p} F_{r}(z, u, v)}{p !\left(\begin{array}{l}
n \\
p
\end{array}\right)\left[z^{n}\right] T_{r}(z)} \sim \frac{2 m^{2 p-3}}{n^{p-1}(p-2) !}\left(\frac{\sigma_{r}}{\sqrt{2}}\right)^{2(p-1)} e^{-\frac{\sigma_{r}^{2} m^{2}}{2 n}} .
$$

Again by setting $m=x \sqrt{n}+o(\sqrt{n})$ we obtain the second part of Theorem 2 .

\section{The height of the leaves}

Defining the generating functions $A_{r}(z, u, v):=\sum_{n \geq 1} \sum_{j \geq 1} \sum_{m \geq 0} \mathbb{P}\left\{H_{n, j}=m\right\} T_{n}^{[r]} z^{n} u^{j} v^{m}$ one can again show by using (1) the following system of functional equations for $A_{r}(z, u, v)$ :

$$
\begin{aligned}
A_{1}(z, u, v) & =\varphi_{0} z u+\frac{z v\left(\varphi\left(y_{1}(z, u)\right)-\varphi\left(T_{1}(z)\right)\right)}{y_{1}(z, u)-T_{1}(z)} A_{1}(z, u, v), \\
A_{2}(z, u, v) & =\varphi_{0} z u+\frac{z v\left(\varphi\left(y_{2}(z, u)\right)-\varphi\left(T_{2}(z)\right)\right)}{y_{2}(z, u)-T_{2}(z)} A_{2}(z, u, v)+A_{1}(z, u, v), \\
\vdots & \\
A_{r}(z, u, v) & =\varphi_{0} z u+\frac{z v\left(\varphi\left(y_{r}(z, u)\right)-\varphi\left(T_{r}(z)\right)\right)}{y_{r}(z, u)-T_{r}(z)} A_{r}(z, u, v)+A_{r-1}(z, u, v),
\end{aligned}
$$

where the generating functions $y_{r}(z, u)$ are defined by $y_{r}(z, u):=\sum_{n \geq 1} \sum_{m \geq 0} \mathbb{P}\left\{L_{n}=m\right\} T_{n}^{[r]} z^{n} u^{m}$. These generating functions satisfy themselves the following system of functional equations:

$$
\begin{aligned}
y_{1}(z, u) & =\varphi_{0} z(u-1)+z \varphi\left(y_{1}(z, u)\right), \\
y_{2}(z, u) & =\varphi_{0} z(u-1)+z \varphi\left(y_{2}(z, u)\right)+y_{1}(z, u), \\
\vdots & \\
y_{r}(z, u) & =\varphi_{0} z(u-1)+z \varphi\left(y_{r}(z, u)\right)+y_{r-1}(z, u) .
\end{aligned}
$$


It follows immediately from Drmota's studies concerning systems of functional equations (see e. g. Drmota (2004)) that the functions $y_{r}(z, u)$ have in a neighbourhood of $u=1$ the following local expansion around the dominant singularity $\rho_{r}(u)$ :

$$
y_{r}(z, u)=\tau_{r}(u)-\sqrt{\frac{2\left[\varphi_{0}(u-1)+\varphi\left(\tau_{r}(u)\right)+\left(\frac{\partial y_{r-1}}{\partial z}\right)\left(\rho_{r}(u), u\right)\right]}{\varphi^{\prime \prime}\left(\tau_{r}(u)\right)}} \sqrt{1-\frac{z}{\rho_{r}(u)}}+\mathcal{O}\left(1-\frac{z}{\rho_{r}(u)}\right),
$$

and furthermore that the number of leaves $L_{n}$ of a $\{1, \ldots, r\}$-labelled tree follows a Gaussian limit law with mean value $\mathbb{E}\left(L_{n}\right) \sim \kappa_{r} n$, where $\kappa_{r}$ is given by

$$
\kappa_{r}=\frac{\varphi_{0} \rho_{r}+\left(\frac{\partial y_{r-1}}{\partial u}\right)\left(\rho_{r}, 1\right)}{\rho_{r}\left(\varphi\left(\tau_{r}\right)+T_{r-1}^{\prime}\left(\rho_{r}\right)\right)}=\frac{1}{\varphi\left(\tau_{r}\right)+T_{r-1}^{\prime}\left(\rho_{r}\right)} \sum_{l=1}^{r} \frac{\varphi_{0}}{\prod_{s=l}^{r-1}\left(1-\rho_{r} \varphi^{\prime}\left(T_{s}\left(\rho_{r}\right)\right)\right)} .
$$

Equation (14) gives immediately the solution

$$
A_{r}(z, u, v)=\frac{\varphi_{0} z u+A_{r-1}(z, u, v)}{1-v z \frac{\varphi\left(y_{r}(z, u)\right)-\varphi\left(T_{r}(z)\right)}{y_{r}(z, u)-T_{r}(z)}}=\sum_{l=1}^{r} \frac{\varphi_{0} z u}{\prod_{s=l}^{r}\left(1-v f_{s}(z, u)\right)},
$$

where we use the abbreviation

$$
f_{s}(z, u):=z \frac{\varphi\left(y_{s}(z, u)\right)-\varphi\left(T_{s}(z)\right)}{y_{s}(z, u)-T_{s}(z)} .
$$

For our asymptotic evaluations of the coefficients of $A_{r}(z, u, v)$ we use partial fraction expansion and obtain (for $n \rightarrow \infty, j \sim c \kappa_{r} n$ and $m=\mathcal{O}(\sqrt{n})$ ) the asymptotic equivalent

$$
A_{r}(z, u, v) \sim \frac{\varphi_{0} z u}{1-v f_{r}(z, u)} \sum_{l=1}^{r} \frac{\left(f_{r}(z, u)\right)^{r-l}}{\prod_{s=l}^{r-1}\left(f_{r}(z, u)-f_{s}(z, u)\right)},
$$

resp. also

$\left[v^{m}\right] A_{r}(z, u, v) \sim\left(\sum_{l=1}^{r} \frac{\varphi_{0} \rho_{r}}{\prod_{s=l}^{r-1}\left(1-\rho_{r} \varphi^{\prime}\left(T_{s}\left(\rho_{r}\right)\right)\right)}\right)\left(f_{r}(z, u)\right)^{m}=\left(\varphi_{0} \rho_{r}+\left(\frac{\partial y_{r-1}}{\partial u}\right)\left(\rho_{r}, 1\right)\right)\left(f_{r}(z, u)\right)^{m}$.

Extracting coefficients of (17) can now be done similar to Drmota (1994) by using Cauchy's integration formula with a double Hankel contour like integration path. We eventually get

$$
\left[z^{n} u^{j} v^{m}\right] A(z, u, v) \sim \frac{\rho_{r}\left(\varphi\left(\tau_{r}\right)+T_{r-1}^{\prime}\left(\rho_{r}\right)\right) \sigma_{r}^{2} \rho_{r}^{-n} m^{2}}{8 \pi\left(\frac{j}{\kappa_{r}}\right)^{\frac{3}{2}}\left(n-\frac{j}{\kappa_{r}}\right)^{\frac{3}{2}}} \exp \left(-\frac{\sigma_{r}^{2} m^{2} n}{8\left(\frac{j}{\kappa_{r}}\right)\left(n-\frac{j}{\kappa_{r}}\right)}\right) .
$$

Setting

we obtain from (18)

$$
\frac{j}{n}=c \kappa_{r}+o(1), \quad \frac{m}{\sqrt{n}}=x+o(1),
$$

$$
\sqrt{n} \mathbb{P}\left\{H_{n, j}=m\right\} \sim \frac{\sigma_{r}^{3} x^{2}}{4 \sqrt{2 \pi}(c(1-c))^{\frac{3}{2}}} \exp \left(-\frac{\sigma_{r}^{2} x^{2}}{8 c(1-c)}\right) .
$$

Since the right-hand side of (19) is the density function of the Maxwell distribution Theorem 3 is shown.

\section{The distribution of the labels}

Defining the generating functions

$N_{r}\left(z ; v_{1}, \ldots, v_{r}\right):=\sum_{n \geq 1} \sum_{m_{i} \geq 0, \text { for } 1 \leq i \leq r} \mathbb{P}\left\{\left(N_{n}^{[1]}, \ldots, N_{n}^{[r]}\right)=\left(m_{1}, \ldots, m_{r}\right)\right\} T_{n}^{[r]} z^{n} v_{1}^{m_{r}} v_{2}^{m_{r-1}} \cdots v_{r}^{m_{1}}$ we obtain from (1) the following system of functional equations:

$$
\begin{aligned}
N_{1}\left(z ; v_{1}\right) & =z v_{1} \varphi\left(N_{1}\left(z ; v_{1}\right)\right), \\
N_{2}\left(z ; v_{1}, v_{2}\right) & =z v_{2} \varphi\left(N_{2}\left(z ; v_{1}, v_{2}\right)\right)+N_{1}\left(z ; v_{1}\right), \\
& \vdots \\
N_{r}\left(z ; v_{1}, v_{2}, \ldots, v_{r}\right) & =z v_{r} \varphi\left(N_{r}\left(z ; v_{1}, v_{2}, \ldots v_{r}\right)\right)+N_{r-1}\left(z ; v_{1}, v_{2}, \ldots, v_{r-1}\right) .
\end{aligned}
$$


Studying the system (20) gives the following local expansion in a neighbourhood of the dominant singularity $z=\rho_{r}\left(v_{1}, \ldots, v_{r}\right)$ uniformly around $\left(v_{1}, \ldots, v_{r}\right)=(1, \ldots, 1)$ :

$N_{r}\left(z ; v_{1}, \ldots, v_{r}\right)=\tau_{r}\left(v_{1}, \ldots, v_{r}\right)-\sqrt{\frac{2\left(v_{r} \varphi\left(\tau_{r}\left(v_{1}, \ldots, v_{r}\right)\right)+\left(\frac{\partial N_{r}-1}{\partial z}\right)\left(\rho_{r}\left(v_{1}, \ldots, v_{r}\right) ; v_{1}, \ldots, v_{r}\right)\right)}{v_{r} \varphi^{\prime \prime}\left(\tau_{r}\left(v_{1}, \ldots, v_{r}\right)\right)}} \sqrt{1-\frac{z}{\rho_{r}\left(v_{1}, \ldots, v_{r}\right)}+\mathcal{O}\left(1-\frac{z}{\rho_{r}\left(v_{1}, \ldots, v_{r}\right)}\right)}$.

Theorem 4 follows now by singularity analysis and applying a theorem of Bender and Richmond (1983).

\section{References}

E. A. Bender and L. B. Richmond. Central and local limit theorems applied to asymptotic enumeration II: Multivariate generating functions. Journal of Combinatorial Theory, Series A, 34:255-265, 1983.

J. Blieberger. Monotonically labelled Motzkin trees. Discrete Applied Mathematics, 18:9-24, 1987.

M. Drmota. The height distribution of leaves in rooted trees. Discrete Mathematics and Applications, 4: 45-58, 1994.

M. Drmota. Combinatorics and asymptotics on trees. Cubo Journal, 6:105-136, 2004.

P. Flajolet and A. M. Odlyzko. Singularity analysis of generating functions. SIAM Journal on Discrete Mathematics, 3:216-240, 1990.

B. Gittenberger. On the contour of random trees. SIAM Journal on Discrete Mathematics, 12:434-458, 1999.

P. Kirschenhofer. On the average shape of monotonically labelled tree structures. Discrete Applied Mathematics, 7:161-181, 1984.

A. Meir and J. W. Moon. On the altitude of nodes in random trees. Canadian Journal of Mathematics, 30:997-1015, 1978

K. Morris and H. Prodinger. Monotone tree structures revisited. Manuscript, 2005.

A. Panholzer. The distribution of the size of the ancestor-tree and of the induced spanning subtree for random trees. Random Structures and Algorithms, 25:179-207, 2004.

H. Prodinger and F. J. Urbanek. On monotone functions of tree structures. Discrete Applied Mathematics, 5:223-239, 1983. 